Louisiana State University

LSU Digital Commons

Faculty Publications

Department of Physics \& Astronomy

$1-28-2005$

\title{
Amplitude and phase control of attosecond light pulses
}

\author{
Rodrigo López-Martens \\ Lunds Tekniska Högskola \\ Katalin Varjú \\ Lunds Tekniska Högskola \\ Per Johnsson \\ Lunds Tekniska Högskola \\ Johan Mauritsson \\ Lunds Tekniska Högskola \\ Yann Mairesse \\ Service des Photons Atomes et Molécules
}

See next page for additional authors

Follow this and additional works at: https://digitalcommons.Isu.edu/physics_astronomy_pubs

\section{Recommended Citation}

López-Martens, R., Varjú, K., Johnsson, P., Mauritsson, J., Mairesse, Y., Salières, P., Gaarde, M., Schafer, K., Persson, A., Svanberg, S., Wahlström, C., \& L'Huillier, A. (2005). Amplitude and phase control of attosecond light pulses. Physical Review Letters, 94 (3) https://doi.org/10.1103/PhysRevLett.94.033001

This Article is brought to you for free and open access by the Department of Physics \& Astronomy at LSU Digital Commons. It has been accepted for inclusion in Faculty Publications by an authorized administrator of LSU Digital Commons. For more information, please contact ir@lsu.edu. 


\section{Authors}

Rodrigo López-Martens, Katalin Varjú, Per Johnsson, Johan Mauritsson, Yann Mairesse, Pascal Salières, Mette B. Gaarde, Kenneth J. Schafer, Anders Persson, Sune Svanberg, Claes Göran Wahlström, and Anne L'Huillier 


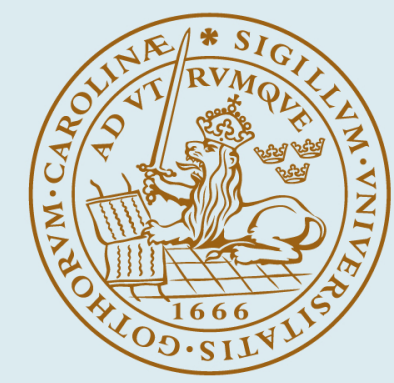

\section{LUND UNIVERSITY}

\section{Amplitude and phase control of attosecond light pulses}

Lopez, Rodrigo; Varju, Katalin; Johnsson, Per; Mauritsson, J; Mairesse, Y; Salieres, P; Gaarde, M B; Schafer, K J; Persson, Anders; Svanberg, Sune; Wahlström, Claes-Göran; L'Huillier, Anne

Published in:

Physical Review Letters

DOI:

10.1103/PhysRevLett.94.033001

2005

Link to publication

Citation for published version (APA):

Lopez, R., Varju, K., Johnsson, P., Mauritsson, J., Mairesse, Y., Salieres, P., Gaarde, M. B., Schafer, K. J., Persson, A., Svanberg, S., Wahlström, C-G., \& L'Huillier, A. (2005). Amplitude and phase control of attosecond light pulses. Physical Review Letters, 94(3). https://doi.org/10.1103/PhysRevLett.94.033001

\section{Total number of authors:}

12

\footnotetext{
General rights

Unless other specific re-use rights are stated the following general rights apply:

Copyright and moral rights for the publications made accessible in the public portal are retained by the authors and/or other copyright owners and it is a condition of accessing publications that users recognise and abide by the legal requirements associated with these rights.

- Users may download and print one copy of any publication from the public portal for the purpose of private study or research.

- You may not further distribute the material or use it for any profit-making activity or commercial gain

- You may freely distribute the URL identifying the publication in the public portal
}

Read more about Creative commons licenses: https://creativecommons.org/licenses/

Take down policy

If you believe that this document breaches copyright please contact us providing details, and we will remove access to the work immediately and investigate your claim. 


\title{
Amplitude and Phase Control of Attosecond Light Pulses
}

\author{
Rodrigo López-Martens, ${ }^{1, *}$ Katalin Varjú, ${ }^{1, \dagger}$ Per Johnsson, ${ }^{1}$ Johan Mauritsson, ${ }^{1,}$ Yann Mairesse, ${ }^{2}$ Pascal Salières, ${ }^{2}$ \\ Mette B. Gaarde, ${ }^{3}$ Kenneth J. Schafer, ${ }^{3}$ Anders Persson, ${ }^{1}$ Sune Svanberg, ${ }^{1}$ Claes-Göran Wahlström, ${ }^{1}$ and Anne L'Huillier ${ }^{1}$ \\ ${ }^{1}$ Department of Physics, Lund Institute of Technology, P. O. Box 118, SE-221 00 Lund, Sweden \\ ${ }^{2}$ Commissariat à l'Energie Atomique, DRECAM/SPAM, Centre d'Etudes de Saclay, 91191 Gif-sur-Yvette, France \\ ${ }^{3}$ Department of Physics and Astronomy, Louisiana State University, Baton Rouge, Louisiana 70803-4001, USA
}

(Received 9 July 2004; published 26 January 2005)

\begin{abstract}
We report the generation, compression, and delivery on target of ultrashort extreme-ultraviolet light pulses using external amplitude and phase control. Broadband harmonic radiation is first generated by focusing an infrared laser with a carefully chosen intensity into a gas cell containing argon atoms. The emitted light then goes through a hard aperture and a thin aluminum filter that selects a 30-eV bandwidth around a $30-\mathrm{eV}$ photon energy and synchronizes all of the components, thereby enabling the formation of a train of almost Fourier-transform-limited single-cycle 170 attosecond pulses. Our experiment demonstrates a practical method for synthesizing and controlling attosecond waveforms.
\end{abstract}

DOI: 10.1103/PhysRevLett.94.033001

PACS numbers: $42.65 . \mathrm{Ky}, 32.80 . \mathrm{Rm}$

More than ten years ago, it was realized that the broadband harmonic emission that occurs when an atom is ionized in an intense infrared laser field [1] can be a potential source of attosecond pulses [2-4]. The characteristic plateau region of the harmonic spectrum [see Fig. 1(a)] spans from the ultraviolet into the soft $\mathrm{x}$-ray region, thus in principle providing enough bandwidth to produce pulses as short as a few tens of attoseconds. However, the duration of the harmonic emission is not as short as its spectral bandwidth would allow [5]. Therefore, controlling the amplitude and phase of the collected harmonic radiation is the biggest obstacle to successfully produce extremely short attosecond pulses. Recently, several pioneering experiments have demonstrated that the selection of individual portions of the broad harmonic spectrum can lead to the emission of attosecond pulses [6-11]. Isolated pulses were produced by Hentschel and collaborators via spectral selection of the few harmonics in the cutoff region that are in phase [7-9,12]. In this work, we generate, compress, and deliver on target 170 attosecond pulses through careful amplitude and phase control of ten consecutive plateau harmonics generated in a laser-driven ionization process and spanning a total bandwidth of $30 \mathrm{eV}$. These pulses consist of only 1.2 periods of the central radiation frequency (30-eV photon energy).

The temporal structure of the high-order harmonics originates from the nontrivial electron dynamics responsible for their emission. When an intense laser field interacts with an atom, the electric field can be sufficiently strong for an electron to be released into the continuum via tunnel ionization. This happens for a range of times within the field cycle and the exact time of release determines the initial condition $[13,14]$ for the electron's trajectory in the continuum. After acceleration in the laser field, the electron can return to the ion core and recombine, leading to the emission of a broadband extreme-ultraviolet (XUV) photon. Both the energy and phase of the emitted light are correlated to the return energy and return time of the electron. The periodicity of this process, twice per cycle, leads in the spectral domain to a series of peaksthe odd harmonics - separated by twice the laser frequency. The first obstacle to generating short attosecond pulses is that each harmonic can be produced in at least two

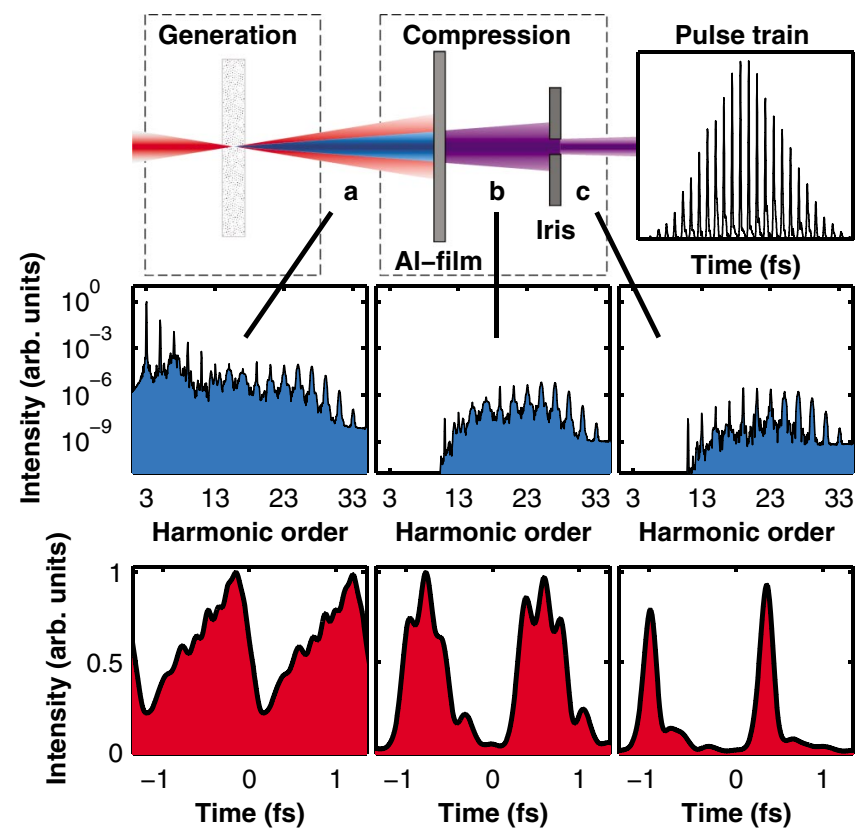

FIG. 1 (color). Principle of the experiment. The generated XUV radiation is compressed in two steps: amplitude and phase control using a $600 \mathrm{~nm}$-thick aluminum film; spatial filtering with a $1.6 \mathrm{~mm}$ iris located $35 \mathrm{~cm}$ after the harmonic generation cell. The spectral and temporal evolution of the XUV radiation, simulated by integrating the time-dependent Schrödinger equation and by solving the Maxwell wave equation for the generated fields, illustrates these effects for the three indicated regions (a)(c). The generated attosecond pulse train is indicated at the top. 
ways, corresponding to two different electron trajectories with the same return energy [15-17]. These two classes of trajectories lead to completely different phase behaviors for the generated fields, thereby preventing the formation of bandwidth-limited XUV pulses. Furthermore, even considering just a single class of trajectories, the emitted XUV radiation is intrinsically frequency chirped. A recent experimental study [5] of plateau harmonics generated in argon and neon shows that, for the shortest trajectories, the attosecond pulses produced indeed exhibit a positive chirp. Without external phase compensation, the useful bandwidth over which the shortest pulses can be obtained is reduced to only a few harmonics.

In the optical domain, many techniques have been developed to reshape the amplitude and rephase the constituent frequencies of ultrashort light pulses in order to keep them localized in time inside the target chamber [18]. The extension of these techniques to the XUV region is a formidable challenge. Freestanding metallic filters [7-10] and multilayer mirrors [7-9] have been used to achieve some spectral filtering in the XUV. The use of plasma media [5] and metallic films [5,19] has been suggested as a way to compensate for the chirp of the XUV radiation. In the present experiments, a 1-mJ-40-fs-800-nm Ti:sapphire laser beam is focused into a windowless gas cell filled with argon to a static pressure of $15 \mathrm{mbar}$ (Fig. 1). The intensity is estimated to be $1.4 \times 10^{14} \mathrm{~W} \mathrm{~cm}^{-2}$. The negative group delay dispersion (GDD) of a thin aluminum foil is used to compensate for the intrinsic chirp across a bandwidth encompassing ten harmonics generated in argon. The onset of transmission of aluminum at lower frequencies and the spectral cutoff of high-harmonic emission at higher frequencies act together as a bandpass filter, which selects a spectrum centered at $30 \mathrm{eV}$ with a total bandwidth of $30 \mathrm{eV}$. To solve the problem of the multiple trajectories contributing to each harmonic, we take advantage of the fact that they have very different coherence properties. The long trajectories lead to spectrally broad and spatially divergent radiation [20,21] and can therefore be removed by adding a small aperture after the harmonic generation cell. Over a spectral range corresponding to the 17 th to the 27 th harmonic frequencies ( $25 \mathrm{to} 40 \mathrm{eV}$ ), aluminum has an almost constant negative GDD, which means that lower frequencies travel more slowly through the foil than the higher frequencies [5,19]. By choosing the appropriate foil thickness, the transmitted harmonics in this spectral range become synchronized. Closer to the absorption edge of aluminum, at $15 \mathrm{eV}$, the GDD varies rapidly and thus is not suitable for chirp compensation below the 17th harmonic. However, in this range, the filter is strongly absorbent and suppresses all of these lower harmonics. In addition to removing the fundamental laser pulse, the $\mathrm{Al}$ filter thus exerts both amplitude and phase control.

To achieve single-cycle attosecond pulses, we have to synchronize more than the six harmonics controlled by the filter. We therefore choose the atomic gas and the generat- ing infrared laser intensity so as to make the high-harmonic spectral cutoff, where the harmonics are naturally well synchronized [9], coincide with the region above $40 \mathrm{eV}$ where the GDD of aluminum is zero. Thus, the internal (atomic) conditions for harmonic generation are tuned to exactly match the effects of the external elements (filter and aperture) located after the generation point, leading to almost total control over the whole span of the broadband XUV spectrum (approximately $30 \mathrm{eV}$ or $7 \mathrm{PHz}$ ). This allows us to shape the harmonic spectrum and synchronize its constituent frequencies to produce a pulse train containing almost transform-limited 170 attosecond bursts, corresponding to only little more than one oscillation of the central carrier frequency around $7 \mathrm{PHz}$.

Numerical simulations of the spectral and temporal structure of the emitted light in our experimental conditions are shown immediately below the schematic experimental setup in Fig. 1. The generated spectrum [Fig. 1(a)] exhibits characteristic high-harmonic behavior, starting with a rapid decrease followed by a plateau region and an abrupt cutoff. The temporal structure of the generated harmonic emission around the top of the laser pulse is dominated by the beating of the two strongest harmonics in the spectrum, the third and the fifth. The pulses transmitted through the $\mathrm{Al}$ filter are still long due to the contributions from the different quantum paths [Fig. 1(b)]. After the spatial filtering, there is only one short singlecycle attosecond burst per half cycle of the driving laser frequency [Fig. 1(c)]. Correspondingly, the individual harmonic peaks are sharper, with strongly suppressed side structures.

To characterize our attosecond pulses, we use the technique called RABITT (reconstruction of attosecond beating by interference of two-photon transitions) [6,22], based on two-photon two-color ionization by one XUV photon and one infrared laser photon as illustrated in Fig. 2. The modulation of the "sideband" signal, originating from the interference between two processes-absorption of an XUV photon and an IR photon $\left(q \omega_{\mathrm{IR}}+\omega_{\mathrm{IR}}\right)$, and absorption of an XUV photon and emission of an IR photon $\left((q+2) \omega_{\mathrm{IR}}-\omega_{\mathrm{IR}}\right)$-is measured as a function of the temporal delay, $\tau$, between the XUV and IR. The signal is proportional to $\cos \left(\phi_{q+2}-\phi_{q}+2 \omega_{\mathrm{IR}} \tau\right)$, allowing us to determine the phase difference $\phi_{q+2}-\phi_{q}$ and thus the delay $\left(\phi_{q+2}-\phi_{q}\right) / 2 \omega_{\mathrm{IR}}$. In our experiment, we recombine both XUV and IR pulses after the generation region, in a Mach-Zehnder arrangement, which allows us to control the phase and amplitude of the broadband XUV radiation independently of the probe. We obtain the variation of the harmonic delay by measuring the spectral phase differences between the harmonics. Combining this knowledge with the easily obtainable harmonic amplitudes enables us to accurately reconstruct the average attosecond pulse in the train. In contrast with previous RABITT measurements $[5,6,11]$, where no active amplitude shaping was per- 


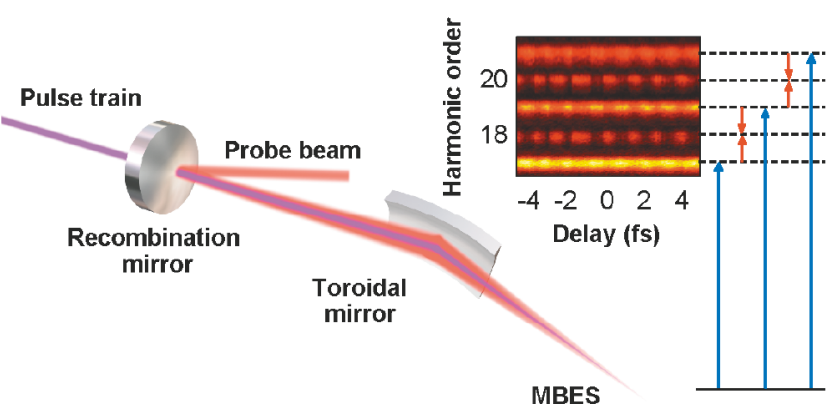

FIG. 2 (color). Attosecond pulse characterization. The measurement, known as RABITT, is based on the two-color twophoton ionization process pictured on the right. A typical acquisition with harmonic and sideband signals (in false colors) is shown as a function of the delay. The IR pulse is overlapped with the XUV pulse by means of a convex spherical mirror with a hole in the center. The curvature and position of this mirror is chosen so that its virtual focal point coincides with the point of harmonic generation, allowing us to match the divergence of the XUV and IR beams. Both beams are refocused by a toroidal platinum mirror into the sensitive region of a magnetic bottle electron time-of-flight spectrometer filled with argon or neon up to a static pressure of $\sim 10^{-4}$ mbar.

formed and the spectral bandwidth was artificially selected by the analysis, we here characterize all the light that reaches the detection chamber. This requires the use of two different target gases with different ionization potentials: argon, which has a lower ionization potential, is used for the lower harmonics and neon, which has a flat ionization cross section over a larger energy range, is used to characterize the highest harmonics.

The amplitude and phase control exerted over the XUV radiation, resulting in compression of the individual attosecond pulses, is demonstrated in Fig. 3. We performed measurements using up to three aluminum films, each $200 \mathrm{~nm}$ thick. The effect of the amplitude shaping [Fig. 3(a)] is mainly to suppress the lower harmonics, as seen by comparing their relative strengths in the two spectra. The relative delay of the harmonics is presented in Fig. 3(b). Between the 17th and 27th harmonic frequencies, the variation of the delay is clearly smaller when using three films, since the aluminum GDD compensates for the delays between the consecutive harmonics. Harmonics higher than the 27th are already well synchronized since the generation intensity was chosen such that these harmonics belong to the cutoff region in argon and their relative phases are hardly affected by the filter. The reconstructed average attosecond pulse shape in the different cases is shown in Fig. 3(c). When no filters are present, we calculate a pulse duration of 480 as assuming the same spectral bandwidth as in the one-filter case. In the threefilter case, we obtain a full width at half maximum of 170 as, the shortest pulse reported to date. At this photon energy $(30 \mathrm{eV})$, this duration corresponds to only 1.2 light cycles.
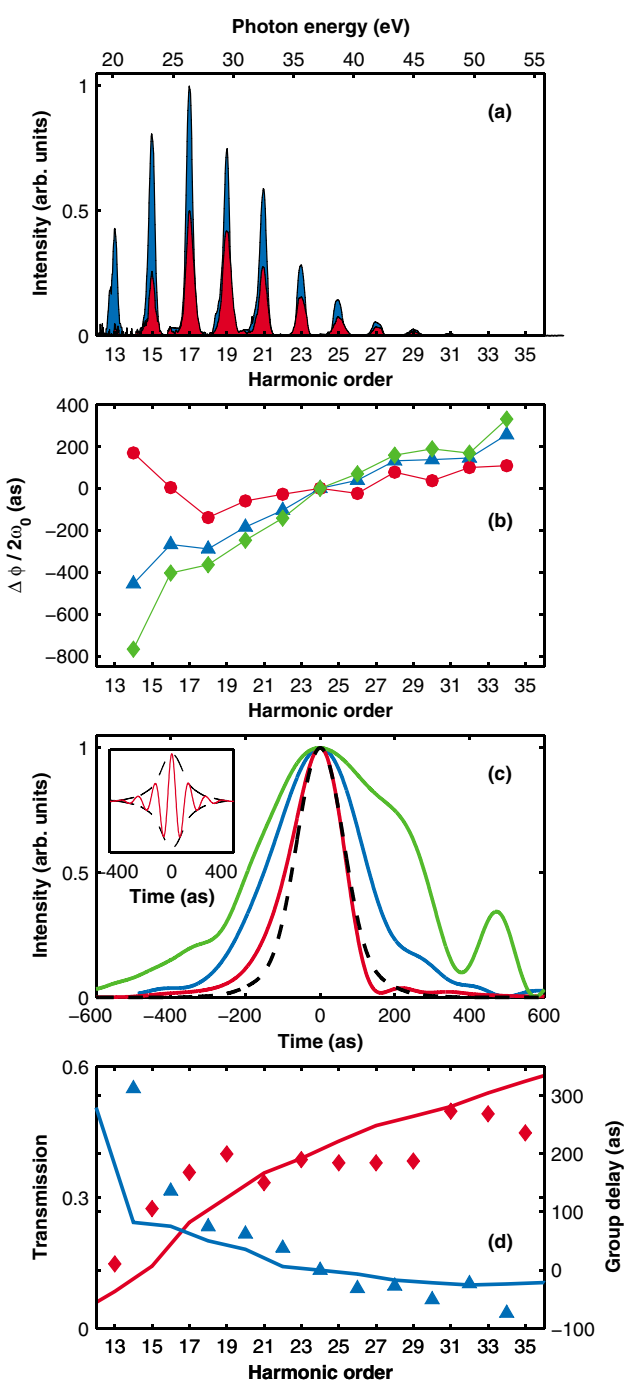

FIG. 3 (color). Compression of XUV radiation via amplitude and phase control. The effect of the aluminum films on the intensities and the synchronization of the harmonics is presented in (a) and (b) for one (blue) and three (red) $200 \mathrm{~nm}$-thick films. These results contain sufficient information to reconstruct the average attosecond pulse in the train (c). The green line shows an extrapolation to zero films assuming the same bandwidth as in the one-filter case. The aluminum improves the synchronization of the harmonics resulting in a compression of the pulse duration from 480 as (zero filter, green curve) down to 280 as (one filter, blue curve) and 170 as (three filters, red curve). The latter is very close to the transform limit of 150 as shown by the dashed line and corresponds to only 1.2 cycles of the carrier frequency (7 PHz). The inset shows the XUV electric field assuming a cosine carrier. The measured transmission (red symbols) and group delay (blue symbols) of our filter are compared to tabulated values (solid lines) in (d).

The transmission and group delay of our aluminum filters, which include a thin layer of oxide with a total thickness estimated at $15 \mathrm{~nm}$, can be extracted from these measurements and compared to tabulated data $[23,24]$. The tabulated transmission data [Fig. 3(d), red line] compare 
very well with those measured for the average filter used in the experiment. The phase difference measured by the RABITT technique [blue symbols in Fig. 3(d)] is reasonably close to the group delay caused by the aluminum foil (with the oxide layer). There is, however, a deviation at low energies (below $25 \mathrm{eV}$ ), which may be due to uncertainty in the refractive indices in this spectral region. Using our experimental results, we can also calculate the intrinsic second-order spectral phase in the plateau region. We obtain $2.1 \times 10^{-32} \mathrm{~s}^{2}$, in agreement with previous measurements [5]. The corresponding chirp rate is $3.7 \times 10^{31} \mathrm{~s}^{-2}$.

In conclusion, we can routinely generate a wellcharacterized train of ultrashort attosecond pulses using a commercial 2-mJ-40-fs-1-kHz Titanium Sapphire laser system, thereby showing that the attosecond time scale can be easily accessed in many laboratories. In contrast to the 130 as-pulse width mentioned in [5], the 170 as measured in the present work does not require further spectral selection: we really produce a train of attosecond pulses. We achieve significantly shorter pulse duration than in [10], thanks to the external phase and amplitude control. We create attosecond pulses in a different energy region than [7-9] and in a technologically simpler way, albeit in a train rather than as an isolated pulse. These results prove that attosecond optical manipulation experiments are indeed possible with currently available technology. This opens the door to the generation of well-characterized attosecond pulses in the soft x-ray region below the sub100 as mark, which are essential for monitoring fast electronic processes in atoms and molecules.

Our technique, combining spatial filtering with amplitude and phase control of the XUV radiation, can be extrapolated to photon energies reaching the soft $\mathrm{x}$-ray region [25], provided lighter rare gases (e.g., neon or helium) or ions [26] are used for the generation of the high harmonics and other materials with negative dispersion are used for the spectral filtering and compression $[5,19]$. The dispersion of atoms is always negative after absorption edges, and the chirp of the pulses in the train is always positive when the short trajectory is selected, making phase control in principle possible over a wide range of XUV energies. Trains of such extremely short pulses will make it possible to study and control a large number of atomic and molecular strong-field processes with unprecedented time resolution [27]. Finally, the manipulations of the XUV amplitudes and phases that we performed in order to reach the single cycle are the first step towards the production of arbitrary attosecond waveforms, which will facilitate broadband coherent control in the XUV range.
We acknowledge the support of the European Community's Improving Human Potential Programme (ATTO), the Knut and Alice Wallenberg Foundation, and the Swedish Science Council. K. S. and M. B. G. acknowledge the support from the National Science Foundation. We acknowledge the help of Ivan Maximov and Zsolt Geretovszky in carrying out scanning electron microscopy studies of the aluminum films at the Solid State Physics Division of Lund University and of Harm Gert Muller and Rob Kemper from the FOM Institute in Amsterdam with the electron spectrometer.

*Current address: Laboratoire d'Optique Appliquée, Palaiseau, France.

${ }^{\dagger}$ On leave from: the Department of Optics and Quantum Electronics, University of Szeged, Szeged, Hungary.

${ }^{\ddagger}$ Current address: Department of Physics and Astronomy, Louisiana State University, Baton Rouge, USA.

[1] M. Ferray et al., J. Phys. B 21, L31 (1988).

[2] G. Farkas and C. Tóth, Phys. Lett. A 168, 447 (1992).

[3] S. E. Harris et al., Opt. Commun. 100, 487 (1993).

[4] P. B. Corkum et al., Opt. Lett. 19, 1870 (1994).

[5] Y. Mairesse et al., Science 302, 1540 (2003).

[6] P. M. Paul et al., Science 292, 1689 (2001).

[7] M. Hentschel et al., Nature (London) 414, 509 (2001).

[8] R. Kienberger et al., Science 297, 1144 (2002).

[9] R. Kienberger, et al., Nature (London) 427, 817 (2004).

[10] P. Tzallas et al., Nature (London) 426, 267 (2003).

[11] S. A. Aseyev et al., Phys. Rev. Lett. 91, 223902 (2003).

[12] I. P. Christov, M. M. Murnane, and H. C. Kapteyn, Phys. Rev. Lett. 78, 1251 (1997).

[13] K. J. Schafer et al., Phys. Rev. Lett. 70, 1599 (1993).

[14] P. B. Corkum, Phys. Rev. Lett. 71, 1994 (1993).

[15] M. Lewenstein et al., Phys. Rev. A 52, 4747 (1995).

[16] P. Antoine et al., Phys. Rev. Lett. 77, 1234 (1996).

[17] M.B. Gaarde and K. J. Schafer, Phys. Rev. Lett. 89, 213901 (2002).

[18] A. M. Weiner, Rev. Sci. Instrum. 71, 1929 (2000).

[19] K. T. Kim et al., Phys. Rev. A 69, 051805(R) (2004).

[20] M. Bellini et al., Phys. Rev. Lett. 81, 297 (1998).

[21] P. Salières et al., Science 292, 902 (2001).

[22] H. G. Muller, Appl. Phys. B 74, S17 (2002).

[23] D. Y. Smith et al., in Handbook of Optical Constants of Solids, edited by E. D. Palik (Academic Press, Orlando, 1985), pp. 369-398.

[24] F. Gervais, in Handbook of Optical Constants of Solids II, edited by E. D. Palik (Academic Press, San Diego, 1991), pp. 761-775.

[25] E. Seres et al., Phys. Rev. Lett. 92, 163002 (2004).

[26] E. A. Gibson et al., Phys. Rev. Lett. 92, 033001 (2004).

[27] K. J. Schafer et al., Phys. Rev. Lett. 92, 023003 (2004). 